\title{
CANCELLING COMPLEX POINTS IN CODIMENSION TWO
}

\author{
MARKO SLAPAR
}

(Received 31 May 2012; accepted 26 June 2012; first published online 9 August 2012)

\begin{abstract}
A generically embedded real submanifold of codimension two in a complex manifold has isolated complex points that can be classified as either elliptic or hyperbolic. In this paper we show that a pair consisting of one elliptic and one hyperbolic complex point of the same sign can be cancelled by a $C^{0}$ small isotopy of embeddings.
\end{abstract}

2010 Mathematics subject classification: primary 32V40; secondary 32S20.

Keywords and phrases: CR manifolds, complex points.

\section{Introduction}

Let $i: Y \hookrightarrow X$ be a real compact ( $2 n-2)$-dimensional manifold $Y$, smoothly embedded into an $n$-dimensional complex manifold $(X, J)$. For $p \in Y$ the complex space $T_{p}^{\mathbb{C}} Y=$ $T_{p} Y \cap J T_{p} Y$ is the maximal complex subspace of the tangent space $T_{p} Y$. In a generic situation, only finitely many points are complex points, meaning that $T_{p} Y=T_{p}^{\mathbb{C}} Y$, and for all other points, the dimension of $T_{p}^{\mathbb{C}} Y$ equals $n-2$. If $Y$ is an oriented manifold and $p \in Y$ is a complex point, the orientation of $T_{p} Y$ can be compared with the induced orientation of $T_{p} Y$, seen as a complex subspace of $T_{p} X$. If these two orientations agree, the complex point is called positive, if not, negative. In some local holomorphic coordinates, defined in a neighbourhood $U$ of an isolated complex point $p \in Y$, the manifold $Y$ can be written as

$$
w=\bar{z}^{T} A z+\operatorname{Re}\left(z^{T} B z\right)+o\left(|z|^{2}\right),
$$

where $(z, w)$ are coordinates in $\mathbb{C}^{n}=\mathbb{C}^{n-1} \times \mathbb{C}, z=\left(z_{1}, z_{2}, \ldots, z_{n-1}\right)$, and $A, B$ are $(n-1) \times(n-1)$ complex matrices, $B=B^{T}$. See [1]. It is easy to check that the value of the determinant of

$$
\left[\begin{array}{ll}
A & \bar{B} \\
B & \bar{A}
\end{array}\right]
$$

Supported by the research program P1-0291 at the Slovenian Research Agency.

(C) 2012 Australian Mathematical Publishing Association Inc. 0004-9727/2012 \$16.00 
is always real, and we call the complex point nondegenerate if this value is nonzero. For generic embeddings, all complex points are nondegenerate and thus isolated. Depending on the sign of the determinant of (1.2), we call the complex point elliptic (positive sign) or hyperbolic (negative sign). It turns out that this definition is independent with respect to biholomorphic coordinate change; see [1, Section 3] or [12]. Note that the terms elliptic and hyperbolic here are used differently by Dolbeault et al. in $[2,3]$. The main result of this paper is the following theorem.

Theorem (Cancellation theorem). Let $i: Y \hookrightarrow X$ be a real compact (2n-2)dimensional manifold $Y$, smoothly embedded into an $n$-dimensional complex manifold $X$. We assume that the embedding is generic, so that complex points are isolated. Let $p, q \in Y$ be a pair of complex points, one elliptic and one hyperbolic, and let $\gamma \subset Y$ be an orientation-preserving smooth arc connecting $p$ and $q$. Let $d$ be some metric on $X$. Then for any neighbourhood $U$ of $\gamma$ and any $\varepsilon>0$, there exists an isotopy $i_{t}: Y \times[0,1] \rightarrow X$ of embeddings so that:

(i) $i_{0}=i$ and $i_{t}(y)=i(y)$ for every $y \in Y \backslash U$;

(ii) $d\left(i_{t}(y), i(y)\right)<\varepsilon$ for every $y \in Y$;

(iii) the set of complex points of $i_{1}(Y)$ equals the set

$$
\{y \in Y \text {; } y \text { is a complex point of } i(Y)\} \backslash\{p, q\} .
$$

The theorem is new for $n \geq 3$. For $n=2$ it was proved by Eliashberg and Harlamov in [4]. The most elegant proof in $n=2$ was given by Nemirovski in [10], but that proof does not seem to adapt nicely to higher dimensions.

If $Y$ is nonorientable, then the obstruction to orientability comes from some handle (or more than one) in a handle decomposition of $Y$. So any two complex points on $Y$ can be connected by an orientation-preserving smooth arc, since, if needed, we can always add an extra loop across such a handle to change the orientation. Using the above theorem, we get the following result.

Corollary 1.1. Let $i: Y \hookrightarrow X$ be a real connected nonorientable $(2 n-2)$-dimensional manifold $Y$, smoothly embedded into an $n$-dimensional complex manifold $X$. Then any pair consisting of an elliptic and a hyperbolic complex point can be cancelled by a $C^{0}$ small isotopy of embeddings.

Now let $Y^{2 n-2} \rightarrow X^{n}$ be an immersion with $Y$ compact and orientable. For immersions, complex points are defined exactly the same as for embeddings, or we can equivalently think of $Y$ as actually being embedded as a zero section in its normal bundle $N Y$. We denote by $e_{ \pm}(Y)$ the number of positive (negative) elliptic complex points on $Y$ and by $h_{ \pm}(Y)$ the number of positive (negative) hyperbolic complex points on $Y$. Let $I_{ \pm}(Y)=e_{ \pm}(Y)-h_{ \pm}(Y)$. The numbers $I_{ \pm}(Y)$ are called Lai indices (see [9]) and can be expressed by the formula

$$
2 I_{ \pm}(Y)=\chi(Y)+\left\langle\sum_{k=0}^{n-1}( \pm 1)^{k+1} e^{k}(N Y) \cup c_{n-1-k}\left(\left.T X\right|_{Y}\right),[Y]\right\rangle
$$


where $\chi(Y)$ is the Euler characteristic of $Y, e(N Y)$ is the Euler class of the normal bundle of $Y \rightarrow X$ and $c_{l}\left(\left.T X\right|_{Y}\right)$ is the $l$ th Chern class of the tangent bundle $T X$ restricted to $Y$. The main theorem has the following corollary.

Corollary 1.2. Let $Y^{2 n-2} \rightarrow X^{n}$ be a real compact oriented manifold of codimension two immersed in a complex manifold $X$. Then the Lai indices $I_{ \pm}$are the only invariants of complex points of $Y$ up to a regular homotopy of immersions.

EXAMPLE. If $Y \subset X$ is a nonsingular compact complex hypersurface in a complex manifold $X$, then after a generic smooth perturbation, it has only isolated complex points. If this perturbation is $C^{1}$ small, then all complex points are positive, since the orientation of the tangent bundle of $Y$ is the induced orientation from $T X$. So

$$
0=2 I_{-}(Y)=\chi(Y)+\left\langle\sum_{k=0}^{n-1}(-1)^{k+1} e^{k}(N Y) \cup c_{n-1-k}\left(\left.T X\right|_{Y}\right),[Y]\right\rangle
$$

This is the adjunction equality for complex hypersurfaces. Let us, for example, consider $Y=\mathbb{C} P^{2}$ as a zero section of a complex line bundle $L \rightarrow \mathbb{C} P^{2}$, and let $L$ correspond to the divisor $d\left[\mathbb{C} P^{1}\right]$. Let $X$ be the total space of $L$. We have

$$
e(N Y)=d P D[\mathbb{C} P 1] \quad \text { and }\left.\quad c_{1}(X)\right|_{Y}=e(N Y)+c_{1}(Y)=(d+3) P D\left[\mathbb{C} P^{1}\right]
$$

where $P D\left[C P^{1}\right]$ is the Poincaré dual of $\left[\mathbb{C} P^{1}\right]$ in $Y$. Since $Y$ is complex, we have $I_{-}(Y)=0$ and

$$
\begin{aligned}
I_{+}(Y) & =\frac{1}{2}\left(\chi(Y)+\left\langle c_{2}(X)+e(N Y) \cup c_{1}(X)+e^{2}(N Y),[Y]\right\rangle\right) \\
& =3+\left\langle e(N Y) \cup c_{1}(X),[Y]\right\rangle=d^{2}+3 d+3 .
\end{aligned}
$$

The smallest value of $I_{+}$is achieved at $d=-1$ and $d=-2$ and is equal to 1 . The first case corresponds to $Y$ being the exceptional hypersurface in the blow-up of a point. So the exceptional $\mathbb{C} P^{2}$ in the blow-up of a complex 3-manifold at a point can be deformed by an isotopy to have only one elliptic positive complex point, and no negative complex points. One can also show this in higher dimensions. When $d=1$, we get $I_{+}=7$. So the standard $\mathbb{C} P^{2} \subset \mathbb{C} P^{3}$ can be deformed to have seven positive complex points, all of them elliptic, and no negative complex points.

\section{Proof of cancellation theorem}

Let $\gamma:[0,1] \rightarrow Y$ be an orientation-preserving arc connecting two isolated complex points $p, q \in Y$. If needed, we can deform the arc slightly so that $p$ and $q$ are the only complex points on $\gamma$. For dimension reasons, we can further assume that for $t \in(0,1)$, $\gamma$ is transverse to $T_{\gamma(t)}^{\mathbb{C}} Y$. The first part of the proof closely follows the first part of the proof of the analogous theorem in dimension two in [6] or in [7, Theorem 9.5.1]. The goal here is to construct an appropriate complex basis of $T X$ in a neighbourhood of $\gamma$, and use it to essentially prescribe a holomorphic diffeomorphism in a neighbourhood 
of $\gamma$ into $\mathbb{C}^{n}$, so that the image of $Y$ becomes a graph over a domain in $\mathbb{C}^{n-1}$. In a neighbourhood of $\gamma$ in $Y$ we choose smooth vector fields $Z_{1}, \ldots, Z_{n-2}, Z_{n-1}, Z$, so that $Z_{1}, \ldots, Z_{n-2}$ form a complex basis for $T_{\gamma(t)}^{\mathbb{C}}$ for every $t \in(0,1), Z_{n-1}$ is tangent to $\gamma$, and $Z_{1}, \ldots, Z_{n-2}, J Z_{1}, \ldots, J Z_{n-2}, Z_{n-1}, Z$ are real independent. We can smoothly extend these vector fields to a neighbourhood of $\gamma$ in $X$. Now let $V$ be any vector field defined in a neighbourhood of $\gamma$ in $X$ that is $\mathbb{C}$ independent of $Z_{1}, Z_{2}, \ldots, Z_{n-1}$. We have

$$
Z=a_{1} Z_{1}+a_{2} Z_{2}+\cdots+a_{n-1} Z_{n-1}+b V
$$

where $a_{1}, a_{2}, \ldots, a_{n-1}, b$ are some complex functions. Along $\gamma$, the only zeros of $b$ are at $p$ and $q$. Since $\gamma$ is orientation-preserving, the orientation of the basis $Z_{1}, J Z_{1}, \ldots, Z_{n-2}, J Z_{n-2}, Z_{n-1}, Z$ compared to the orientation of $Z_{1}, J Z_{1}, \ldots$, $Z_{n-1}, J Z_{n-1}$ must be the same at both $p$ and $q$, implying that $\operatorname{Im} a_{n-1}$ is of the same sign at $p$ and $q$. We can assume that the sign is positive. Now let $W=-i m \bar{b} Z_{n-1}+V$ for some large enough positive $m$. $W$ is again $\mathbb{C}$ independent of $Z_{1}, Z_{2}, \ldots, Z_{n-1}$ and

$$
Z=a_{1} Z_{1}+a_{2} Z_{2}+\cdots+a_{n-2} Z_{n-2}+\left(a_{n-1}+i m|b|^{2}\right) Z_{n-1}+b W .
$$

If $m$ is large enough, $\operatorname{Im}\left(a_{n-1}+i m|b|^{2}\right)$ is positive on all of $\gamma$. On a small neighbourhood $U$ of $\gamma$ in $X$, we can define a diffeomorphism $F: U \rightarrow \mathbb{C}^{n}=$ $\left\{\left(z_{1}, \ldots, z_{n-1}, w\right)\right\}, z_{k}=x_{k}+i y_{k}, w=u+i v$, so that

$$
F(\gamma) \subset\left\{\left(z_{1}, \ldots, z_{n-1}, w\right), z_{1}=\cdots=z_{n-2}=y_{n-1}=w=0\right\}
$$

and $D F\left(Z_{k}\right)=\partial / \partial x_{k}, \quad D F\left(J Z_{k}\right)=\partial / \partial y_{k}, \quad k=1, \ldots, n-1, \quad D F(W)=\partial / \partial u \quad$ and $D F(W)=\partial / \partial v$ along $\gamma$, where $D F$ is the derivative of $F$. The map $F$ is complex linear along $\gamma$ and we have $d v(D F(Z))>0$ along $\gamma$. The projection of $D F(T Y)$ to $T \mathbb{C}^{n-1} \times\{0\}$ is thus surjective near $\gamma$ and the image $F(Y) \in \mathbb{C}^{n}$ can be written as a graph over $\mathbb{C}^{n-1}$ by the implicit function theorem. Due to [11, Theorem 1], we can $C^{1}$ approximate $F$ by a holomorphic map in a small Stein neighbourhood of $\gamma$ in $X$. In a neighbourhood of $\gamma$ the manifold $Y$ can therefore be seen as a graph of a smooth function $f: D \rightarrow \mathbb{C}$ for some domain $D$ in $\mathbb{C}^{n-1}$. Before we continue, let us show the following lemma.

Lemma. Let $f: D \rightarrow \mathbb{C}$ be a smooth function from a domain $D \in \mathbb{C}^{n}$. Then $(a, f(a))$ is a complex point of the graph of $f$ in $\mathbb{C}^{n+1}$ if and only if $\bar{\partial} f(a)=0$. Such a complex point is elliptic if and only if the section $\bar{\partial} f$ of $T^{(0,1)} D=D \times \mathbb{C}^{n}$ has a positive transverse intersection with the zero section at a, and hyperbolic if this intersection is negative.

Proof. The first part is trivial. For the second part, let us assume that $a=0$. Since we assume that $\bar{\partial} f(a)=0$, we can locally near $a$ write $f(z)=b^{T} z+\bar{z}^{T} A z+\frac{1}{2} \bar{z}^{T} \bar{B}_{\bar{z}}+$ $\frac{1}{2} z^{T} C z+o\left(|z|^{2}\right)$, for some $n \times n$ complex matrices $A, B, C$ and some $b \in \mathbb{C}^{n}$. (We can get to the expression (1.1) if we holomorphically change the variable $w$ to $w-b^{T} z-\frac{1}{2}\left(z^{T}(C-B) z\right)$.) Then the section $\bar{\partial} f$ of $T^{(0,1)} D=D \times \mathbb{C}^{n}$ is $\bar{\partial} f=F(z)=$ $A z+\bar{B} \bar{z}+o(|z|)$. To calculate the sign of the intersection with the zero section at 0 , we need to calculate the determinant of the real Jacobian of $F$ at 0 . Let $A=A_{1}+i A_{2}$, 
$B=B_{1}+i B_{2}$ and $z=x+i y$, where $A_{1}, A_{2}, B_{1}, B_{2}$ are real $n \times n$ matrices and $x, y \in \mathbb{R}^{n}$. The Jacobian at 0 equals $\left[\begin{array}{cc}A_{1}+B_{1} & -A_{2}-B_{2} \\ A_{2}-B_{2} & A_{1}-B_{1}\end{array}\right]$. A simple matrix manipulation gives us

$$
\begin{aligned}
\left|\begin{array}{cc}
A_{1}+B_{1} & -A_{2}-B_{2} \\
A_{2}-B_{2} & A_{1}-B_{1}
\end{array}\right| & =\left|\begin{array}{cc}
A+\bar{B} & i A-i \bar{B} \\
A_{2}-B_{2} & A_{1}-B_{1}
\end{array}\right|=\left|\begin{array}{cc}
2 A & i A-i \bar{B} \\
-i A+i B & A_{1}-B_{1}
\end{array}\right| \\
& =\left|\begin{array}{cc}
2 A & -A+\bar{B} \\
-A+B & A_{1}-B_{1}
\end{array}\right|=\left|\begin{array}{cc}
2 A & -A+\bar{B} \\
B & \frac{1}{2} \bar{A}-\frac{1}{2} B
\end{array}\right| \\
& =\left|\begin{array}{cc}
A & -A+\bar{B} \\
B & \bar{A}-B
\end{array}\right|=\left|\begin{array}{ll}
A & \bar{B} \\
B & \bar{A}
\end{array}\right| .
\end{aligned}
$$

So the sign of the intersection with the zero section corresponds to the sign of the determinant in the definition of elliptic and hyperbolic points.

We now complete the proof of the theorem. By assumption, the graph of $f: D \rightarrow \mathbb{C}$ has one elliptic and one hyperbolic point. Using the previous lemma, the section $\bar{\partial} f$ intersects the zero section at two points of opposite sign. The intersections can thus be removed using the Whitney trick, so we can add a smooth $(0,1)$ form $\alpha$, compactly supported in $D$, so that $\bar{\partial} f+\alpha$ has no zeros on $D$. Let $G: D \rightarrow \mathbb{C}^{n}$ be the map $G(z)=(z, f(z))$. The derivative

$$
D G=\left(d z_{1}, d z_{2}, \ldots, d z_{n-1}, d f\right): T D=D \times \mathbb{C}^{n-1} \rightarrow T C^{n}=\mathbb{C}^{n} \times \mathbb{C}^{n}
$$

can be deformed by a homotopy $H_{t}=\left(d z_{1}, d z_{2}, \ldots, d z_{n-1}, d f+t \alpha\right)$ covering the embedding $F$. Since $\bar{\partial} f+\alpha$ is never zero on $D$, the end map $H_{1}=$ $\left(d z_{1}, d z_{2}, \ldots, d z_{n-1}, \partial f+\bar{\partial} f+\alpha\right)$ never maps $T_{z} D$ to a complex subspace of $T_{F(z)} \mathbb{C}^{n}$. We can apply the relative version of the h-principle of Gromov for co-real embeddings, [8] or [5], to get an embedding $F_{1}: D \rightarrow \mathbb{C}^{n}$ that is $C^{0}$ close to $F$ and agrees with $F$ near the boundary of $D$. This completes the proof.

\section{References}

[1] A. Coffman, 'CR singularities of real fourfolds in $C^{3}$, Illinois J. Math.(3) 53 (2009), 939-981.

[2] P. Dolbeault, G. Tomassini and D. Zaitsev, 'On boundaries of Levi-flat hypersurfaces in $C^{n}$ ', C. $R$. Math. Acad. Sci. Paris 341(6) (2005), 343-348.

[3] P. Dolbeault, G. Tomassini and D. Zaitsev, 'On Levi-flat hypersurfaces with prescribed boundary', Pure Appl. Math. Q. 6(3) (2010), 725-753, Special issue in honor of Joseph J. Kohn. Part 1.

[4] Y. Eliashberg and V. M. Harlamov, 'On the number of complex points of a real surface in a complex surface', Proc. Leningrad Int. Topol. Conf. (1982), 143-148.

[5] Y. Eliashberg and N. Mishachev, Introduction to the h-principle, Graduate Studies in Mathematics, 48 (American Mathematical Society, Providence, RI, 2002).

[6] F. Forstnerič, 'Complex tangents of real surfaces in complex surfaces', Duke Math. J. 67(2) (1992), 353-376.

[7] F. Forstnerič, Stein Manifolds and Holomorphic Mappings, Ergebnisse der Mathematik und ihrer Grenzgebiete (3), 56 (Springer, Berlin, 2011).

[8] M. Gromov, Partial Differential Relations, Ergebnisse der Mathematik und ihrer Grenzgebiete (3), 9 (Springer, Berlin, 1986). 
[9] H. F. Lai, 'Characteristic classes of real manifolds immersed in complex manifolds', Trans. Amer. Math. Soc. 172 (1972), 1-33.

[10] S. Nemirovski, 'Complex analysis and differential topology on complex surfaces', Uspekhi Math. Nauk. 45(4) (1999), 47-74.

[11] R. M. Range and Y. T. Siu, ' $C^{k}$ approximation by holomorphic functions and $\bar{\partial}$-closed forms on $C^{k}$ submanifolds of a complex manifold', Math. Ann. 210 (1974), 105-122.

[12] M. Slapar, 'Modeling complex points up to isotopy', J. Geom. Anal., to appear, doi:10.1007/s12220-012-9312-6.

MARKO SLAPAR, Faculty of Education, University of Ljubljana, Kardeljeva Ploščad 16, 1000 Ljubljana, Slovenia

and

Institute of Mathematics, Physics and Mechanics, Jadranska 19, 1000 Ljubljana, Slovenia

e-mail: marko.slapar@pef.uni-lj.si 